\title{
Patient Denialism for COVID19 Pose Difficulty for Treating Physicians
}

\section{Rizwan Pervaiz, ${ }^{1}$ Muhammad Asim Rana, ${ }^{2}$ Mohammad Ahad Qayyum, ${ }^{3}$ Ali Munawar, ${ }^{4}$ Sana Tariq, ${ }^{5}$ Abdullah Ali Lashari ${ }^{6}$}

\begin{abstract}
Objectives: As patients refusal has been a major issue in diagnosing the disease, we carried out this study to determine the magnitude of the refusal or denial of suspected COVID 19 cases.

Methods: All patients seen with COVID 19 symptoms in medical out doors and emergency in Bahria International Hospital, Lahore in two weeks period from April 15, 2020 to April 30, 2020 were included in study. COVID 19 symptoms were ascertained on WHO guidelines labelling patients as COVID 19 suspected through laboratory tests. Depending upon results individual consultation with each patient was arranged to convince them to carry out nasopharyngeal swab for SARS Co-V-2 PCR. Some patients refused despite counselling for follow up regarding symptoms and advised tests. The public fear was the main barrier for the suspected cases to get tested. Each patient who refused PCR test was labelled as in denial.

Results: Of the 40 cases diagnosed as suspected COVID 19.21 refused to get tested. Majority (15) were male.Fever with chills was common among all with majority presented with shortness of breath (43\%) followed by diarrhea (33\%) and chest pain (9\%).

Conclusion: Patients who remain in denial pose a great threat to society as by spreading the virus or deteriorating themselves. An effective campaign on government level should be run to overcome the fear which has made this infection a social stigma to help contain this pandemic in a resource scarce country like Pakistan.

Keywords: Coronavirus denial, COVID-19 denial, COVID in Pakistan, Public reaction to COVID 19

How to cite: Pervaiz R., Rana A.M., Qayyum A.M., Munawar A., Tariq S., Lashari A.A. Patient denialism for COVID19 pose difficulty for treating Physicians. Esculapio 2021;17(02):184-187.

DOI: https://doi.org/10.51273/esc21.2517215
\end{abstract}

\section{Introduction}

$\mathrm{W}$ ith the surging rate of spread of the novel Coronavirus-19 around the globe, each country has had to take measures to contain the spread. Most notable is the marked variation in strategies adopted by each individual country ranging from Sweden at one end of spectrum wherein all primary schools and

1. Rizwan Pervaiz

3. Mohammad Ahad Qayyum

5. Sana Tariq

1-2: Department of Internal and Critical Care Medicine, Bahria International Hospital, Lahore, Pakistan

3. Department of Nephrology, division of Medicine, Bahria International Hospital, Lahore, Pakistan

4-5: Department of Internal and Critical Care Medicine, Bahria International Hospital, Lahore, Pakistan

6. Department of Anesthesia and Critical Care, Bahria International Hospital, Lahore, Pakistan

Correspondence:

Dr. Rizwan Pervaiz, Department of Internal and Critical Care Medicine, Bahria International Hospital, Lahore, Pakistan

Email:drrizwanpervaiz@gmail.com

Submission Date:

1st Revision Date:

Acceptance Date:
14-02-2021

04-03-2021

15-04-2021 restaurants remained open as per normal routine to the other end of the spectrum wherein Saudi Arabia imposed a curfew in its cities. ${ }^{1,2}$ Each strategy had its own merits and demerits which are not within the purview of this discussion. However, whatever strategy adopted there has been a variable but consistent mental baggage attached to the COVID-19 pandemic in every country. This mental baggage has ranged from denial to downright major depressive illness. ${ }^{3}$

With the strategies adopted by Pakistan, health professionals at our facility have noted a significant amount of denial in patients suspected to have COVID-19 resulting in an outright refusal to get tested. This denial and refusal to accept their COVID related symptoms and to seek a management plan is a conundrum because at one hand lies the basic human right of each individual over his/her own health while on the other lies the fact that this person could potentially be a health hazard to the general public at large. This study sheds light on the magnitude of the problem whereby patients suspec- 
ted to have COVID 19 refuse to accept standard deviation was calculated for continuous the diagnosis. Furthermore, refuse to get tested to get a confirmed diagnosis.

\section{Methods}

All consecutive patients seen for COVID related symptoms seen in our medical unit over a 2-week period were included in the study. These included patients who were referred from the COVID 19 help desk located at the gate of the hospital, emergency unit as well as those who walked in to receive treatment. The medical specialists ascertained COVID 19 related symptoms based on World Health Organization guidelines; and labeled the patient's status as a "suspected COVID 19 case" as per international definition(4). To further support the suspected diagnosis of a COVID 19 case each patient underwent a hematology and biochemical workup indirectly indicative of COVID 19 which looked for lymphocytopenia, raised $\mathrm{C}$ reactive protein, raised D-dimers, raised ferritin and normal pro-calcitonin levels. ${ }^{5,6}$

Each patient was given a minimum 15 minute consultation with the medical consultant on-call to explain the probable diagnosis and what should be the management plan ahead including diagnostics, treatment option and the possibility of quarantine as per Government of Punjab's instructions. ${ }^{7}$ Only hemodynamically stable patients who did not require inotropic or mechanical ventilation were included in the study. Critical patients requiring admission in the intensive care unit were excluded, as they required mandatory testing.

A patient who refused to get tested despite 15-minute intensive counselling by medical consultant was labeled to be in denial of his/her underlying suspected COVID 19 diagnosis.

\section{Results}

A total of 40 outdoor patients in our medical unit were given a diagnosis of "suspected COVID 19" over the 2 weeks study period. 21 cases out of these 40 remained in denial and refused to get tested by nasopharyngeal RT PCR for COVID 19. Amongst these 21 patients, 15 were male and 6 were female. The average age was 51.4 years (Table $\mathbf{1}$ ).

All patients shared a history of fevers with chills preceding their presenting complaint. The majority of the patients presented with complaints of shortness of breath (43\%) followed by diarrhea (33\%), chest pain $(9 \%)$, and one patient each presented with simply
Table 1: Demographic Data of Patients of Suspected COVID in Denial

\begin{tabular}{|c|c|c|c|}
\hline Male Patients: & 15 & \multirow{2}{*}{$\begin{array}{c}\text { Male: Female } \\
\text { ratio }\end{array}$} & \multirow{2}{*}{$2.5: 1$} \\
\hline Female Patients: & 6 & & \\
\hline Minimum Patient Age: & 83 & \multirow{2}{*}{ Average age: } & \multirow[t]{2}{*}{51.4286} \\
\hline Maximum Patient Age: & 14 & & \\
\hline
\end{tabular}

fever, lethargy or abdominal pain (Fig-1).

\section{Presenting Complaints}

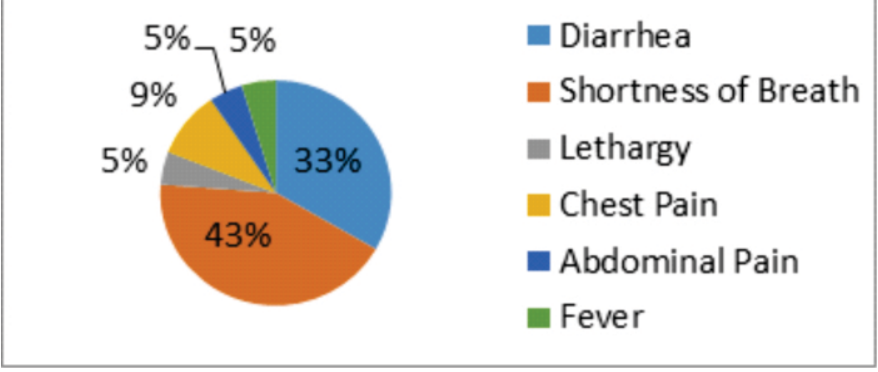

Figure 1: Figurative Description of Major Presenting Complaints of Patients.

The laboratory investigations in each patient included pointed towards the possibility of a COVID 19 infection (Table-1).

Additionally, some laboratory results were strikingly high (D-Dimers with a maximum value of 10000.28, Ferritin at 1207.72 and LDH at 1045).

Table 2: Laboratory Parameters of Suspected COVID Cases Indicating Possibility of COVID

\begin{tabular}{cccc}
\hline Parameter & Major/ Max & Minor/ Min & Average \\
\hline Age & 83 & 14 & 51.43 \\
WCC & 17.6 & 6.37 & 11.46 \\
Lymphos & 48 & 3 & 15.80 \\
CRP & 336.8 & 1.05 & 93.58 \\
D-Dimers & 10000.28 & 159 & 1316.15 \\
LDH & 1045 & 125 & 346.00 \\
Albumin & 4.5 & 0.7 & 3.00 \\
ALT & 2086 & 13 & 184.59 \\
AST & 4078 & 10 & 287.41 \\
Ferritin & 1207.72 & 33.6 & 391.88 \\
Creatinine & 7.2 & 0.5 & 2.13 \\
\hline
\end{tabular}

\section{Discussion}

Biswas, Drogin, \& Gutheilvery prudently stated, "treatment delayed is treatment denied". Although while Biswas et al. were implicating the medical fraternity as the cause of the delay; the current COVID 19 situation in Pakistan seems to suggest that the shoe is on the other foot here with the patients causing a delay in their own treatment. Furthermore, it is not the delay in treatment alone, which is distressing, as the denial creates a barrier 
between treating physician and patient and he/she is not willing to take even the symptomatic treatment and questions all the prescribed drugs anticoagulation for instance in case of raised D-Dimers, but the fact that the patients refusing to get tested or treated in the first place get to roam free in the general public and being in denial do not follow isolation precautions making them a significant health risk. ${ }^{8}$

This refusal to get tested stems from a general sense of denial that he/she has or could possibly have acquired the virus. Personal experiences revealed that when the discussion was opened to the probability of COVID infection and we tried to convince these patients to get their COVID PCR done, all of them denied the possibility of having contracted the virus, and some went on to blame the physicians for "deliberately labeling them as COVID victims". All of these patients lost the follow up after having left the medical facility after this discussion despite being requested to think over their decision and come back.

The distressingly aggressive rate of the spread of the COVID-19 disease due to the novel Coronavirus is almost universal knowledge due to the corroboration and documentation provided by a multitude of international sources. ${ }^{9}$ The virus in question has infected 212 countries and territories, which is over $84 \%$ of all the countries and territories as recognized by the United Nations.

The first two cases of COVID-19 in the Islamic Republic of Pakistan were reported in Karachi, on the 26th of February, 2020, and by the time of writing this paper, May 25th 2020, the number of cases has escalated beyond 56,000 confirmed cases across all the provinces. Over this period of only around 90 days, Pakistan has experienced the loss of 1167 lives. ${ }^{10}$

The most exponential of this increase has been in recent days. Government response has varied over the time period as the information about this novel virus were evolving in an effort to control the disease, but sufficient measures to control the disease have not yet been achieved. Parts of the country have been placed under lockdown and social distancing has been encouraged, as it has been attested as the most efficient way to control the spread of infection. ${ }^{11}$ Unfortunately, the common folk have not all adopted stern social distancing methods due to the social taboo that has surrounded the virus and disease, owing in part to public psychology and in part to culture.

Many people are still in denial of the severity of the disease and its rate of spread, while others ignore their symptoms and avoid being tested. There are several reasons for this, including public fear, religious clergies and poverty. Some religious clergies have denied the gravity of the virus and continued to hold congregations over this period, due to which the people following them have also continued to go about their lives normally without precaution. This has, without a doubt, contributed to the increase in cases.

The people that tend to ignore their symptoms and avoid sharing their concerns have anxieties related to becoming social pariahs and the inability to pay for tests and treatment. The social taboo surrounding the disease and the fear that people have associated with it induces lack of empathy towards infected patients and creates an air of blame surrounding them, as if they are more criminals than chance victims of this plague. This is, quite largely, due to the way patients are handled in government response. People are taken out of their houses to be put in government quarantine unannounced and at all possible hours. Upon returning home after their isolation period, the method of their initial removal has often alienated them from their neighbors, making it difficult for them to fit back into society. ${ }^{12}$

There are also many people, both young and old, who have an unrealistic optimism bias, believing themselves to be more resistant and somehow immune to the disease.

\section{Conclusion}

The data presented in this document is of a small patient group from just one hospital of many in the country. It can be assumed that multiple groups like such exist, and in their refusal of acknowledging their symptoms, they pose a threat to society in the case that they do not self-isolate, as well as to medical staff.

\section{Conflict of Interest None}

\section{References}

1. Team EE. Latest updates on COVID-19 from the European Centre for Disease Prevention and Control. Eurosurveillance. 2020;25(6).

2. Al-Tawfiq JA, Memish ZA. COVID-19 in the Eastern Mediterranean Region and Saudi Arabia: prevention and therapeutic strategies. International Journal of Antimicrobial Agents. 2020;55(5):105968.

3. Torales J, O'Higgins M, Castaldelli-Maia JM, Ventri- 
glio A. The outbreak of COVID-19 coronavirus and its impact on global mental health. International Journal of Social Psychiatry. 2020:0020764020915212.

4. Cascella M, Rajnik M, Cuomo A, Dulebohn SC, Di Napoli R. Features, evaluation and treatment coronavirus (COVID-19). Statpearls [internet]: StatPearls Publishing; 2020.

5. Terpos E, Ntanasis-Stathopoulos I, Elalamy I, Kastritis E, Sergentanis TN, Politou M, et al. Hematological findings and complications of COVID-19. American journal of hematology. 2020.

6. Zhu J, Ji P, Pang J, Zhong Z, Li H, He C, et al. Clinical characteristics of 3,062 COVID-19 patients: a metaanalysis. Journal of Medical Virology. 2020.

7. Sethi BA, Sethi A, Ali S, Aamir HS. Impact of Coronavirus disease (COVID-19) pandemic on health professionals. Pakistan Journal of Medical Sciences. 2020; 36(COVID19-S4).

8. Biswas J, Drogin EY, Gutheil TG. Treatment Delayed is Treatment Denied. J Am Acad Psychiatry Law. 2018; 46:447-53.

9. Organization WH. Naming the coronavirus disease
(COVID-19) and the virus that causes it. 2020. URL https://www.who.int/emergencies/diseases/novelcoronavirus-2019/technical-guidance/naming-thecoronavirus-disease-(covid-2019)-and-the-virusthat-causes-it. 2020.

10. Yousaf M, Zahir S, Riaz M, Hussain SM, Shah K. Statistical analysis of forecasting COVID-19 for upcoming month in Pakistan. Chaos, Solitons \& Fractals. 2020: 109926.

11. Kraemer MU, Yang C-H, Gutierrez B, Wu C-H, Klein B, Pigott DM, et al. The effect of human mobility and control measures on the COVID-19 epidemic in China. Science. 2020;368(6490):493-7.

12. Guan W-j, Ni Z-y, Hu Y, Liang W-h, Ou C-q, He J-x, et al. Clinical characteristics of coronavirus disease 2019 in China. New England journal of medicine. 2020;382(18):1708-20.

\section{Authors Contribution}

R.P: Conceptualization of Project

M.R, M.A.Q: Data Collection

A.M., S.T: Literature Search 